\title{
Fixed points by certain iterative schemes with applications
}

\author{
Ahmed El-Sayed Ahmed ${ }^{1,2^{*}}$ and Sayed Attia Ahmed ${ }^{3,4}$
}

${ }^{\text {*} C o r r e s p o n d e n c e: ~}$

ahsayed80@hotmail.com

1 Department of Mathematics,

Faculty of Science, Sohag University,

Sohag, 82524, Egypt

${ }^{2}$ Mathematics Department, Faculty

of Science, Taif University,

El-Hawiyah, P.O. Box 888, El-Taif,

5700, Saudi Arabia

Full list of author information is

available at the end of the article

\begin{abstract}
The main aim of this paper is to present the concept of general Mann and general Ishikawa type double-sequences iterations with errors to approximate fixed points. We prove that the general Mann type double-sequence iteration process with errors converges strongly to a coincidence point of two continuous pseudo-contractive mappings, each of which maps a bounded closed convex nonempty subset of a real Hilbert space into itself. Moreover, we discuss equivalence from the $S, T$-stabilities point of view under certain restrictions between the general Mann type double-sequence iteration process with errors and the general Ishikawa iterations with errors. An application is also given to support our idea using compatible-type mappings.

MSC: $47 \mathrm{H} 10 ; 54 \mathrm{H} 25$
\end{abstract}

Keywords: fixed points; Hilbert spaces; Mann iteration; pseudo-contractive maps; weakly compatible maps

\section{Introduction}

In the last few decades investigations of fixed points by some iterative schemes have attracted many mathematicians. With the recent rapid developments in fixed point theory, there has been a renewed interest in iterative schemes. The properties of iterations between the type of sequences and kind of operators have not been completely studied and are now under discussion. The theory of operators has occupied a central place in modern research using iterative schemes because of its promise of enormous utility in fixed point theory and its applications. There are a number of papers that have studied fixed points by some iterative schemes (see [1]). It is rather interesting to note that the type of operators play a crucial role in investigations of fixed points.

The Mann iterative scheme was invented in 1953 (see [1-3]), and it is used to obtain convergence to a fixed point for many classes of mappings (see [4-16] and others). The idea of considering fixed point iteration procedures with errors comes from practical numerical computations. This topic of research plays an important role in the stability problem of fixed point iterations. In 1995, Liu [17] initiated a study of fixed point iterations with errors. Several authors have proved some fixed point theorems for Mann-type iterations with errors using several classes of mappings (see [18-28] and others).

Suppose that $H$ is a real Hilbert space and $A$ is a nonlinear mapping of $H$ into itself. The map $A$ is said to be accretive if $\forall x, y \in D(A)$, we have that

$$
\langle A x-A y, x-y\rangle \geq 0
$$

○2014 El-Sayed Ahmed and Ahmed; licensee Springer. This is an Open Access article distributed under the terms of the Creative Commons Attribution License (http://creativecommons.org/licenses/by/2.0), which permits unrestricted use, distribution, and reproduction in any medium, provided the original work is properly cited. 
and it is said to be strongly accretive if $A-k I$ is accretive, where $k \in(0,1)$ is a constant and $I$ denotes the identity operator on $H$.

The map $A$ is said to be $\phi$-strongly accretive if $\forall x, y \in E$, exists a strictly increasing function $\phi:[0, \infty) \rightarrow[0, \infty)$ with $\phi(0)=0$ such that

$$
\langle A x-A y, x-y\rangle \geq \phi(\|x-y\|)\|x-y\|
$$

and it is called uniformly accretive if there exists a strictly increasing function $\psi:[0, \infty) \rightarrow$ $[0, \infty)$ with $\psi(0)=0$ such that $\langle A x-A y, x-y\rangle \geq \psi(\|x-y\|)$.

Let $N(A)=\left\{x^{*} \in H: A x^{*}=0\right\}$ denote the null space (set of zero) of $A$. If $N(A) \neq \phi$ and (1) holds for all $x \in D(A)$ and $y \in N(A)$, then $A$ is said to be quasi-accretive. The notions of strongly, $\phi$-strongly, uniformly quasi-accretive are similarly defined. $A$ is said to be $m$-accretive if $\forall r>0$ the operator $(I+r A)$ is surjective. Closely related to the class of accretive maps is the class of pseudo-contractive maps.

A map $T: H \rightarrow H$ is said to be pseudo-contractive if $\forall x, y \in D(T)$ we have that

$$
\langle(I-T) x-(I-T) y, x-y\rangle \geq 0
$$

observe that $T$ is pseudo-contractive if and only if $A=(I-T)$ is accretive.

A mapping $T: H \rightarrow H$ is called Lipschitzian (or $L$-Lipschitzian) if there exists $L>0$ such that

$$
\|T x-T y\| \leq L\|x-y\|, \quad \forall x, y \in H .
$$

In the sequel we use $L>1$.

Definition 1.1 (see e.g. [24]) Let $\mathbb{N}$ denote the set of all natural numbers, and let $E$ be a normed linear space. By a double sequence in $E$ we mean a function $f: \mathbb{N} \times \mathbb{N} \rightarrow E$ defined by $f(n, m)=x_{n, m} \in E$.

The double sequence $\left\{x_{n, m}\right\}$ is said to converge strongly to $x^{*}$ if for a given $\epsilon>0$, there exist integers $N, M>0$ such that $\forall n \geq N, m \geq M$, we have that

$$
\left\|x_{n, m}-x^{*}\right\|<\epsilon
$$

If $\forall n, r \geq N, m, t \geq M$, we have that

$$
\left\|x_{n, r}-x_{m, t}\right\|<\epsilon
$$

then the double sequence is said to be Cauchy. Furthermore, if for each fixed $n, x_{n, m} \rightarrow x_{n}^{*}$ as $m \rightarrow \infty$ and then $x_{n}^{*} \rightarrow x^{*}$ as $n \rightarrow \infty$, so $x_{n, m} \rightarrow x^{*}$ as $n, m \rightarrow \infty$.

In 2002, Moore [24] introduced the following theorem.

Theorem A Let C be a bounded closed convex nonempty subset of a (real) Hilbert space $H$, and let $T: C \rightarrow C$ be a continuous pseudo-contractive map. Let $\left\{\alpha_{n}\right\}_{n \geq 0},\left\{a_{k}\right\}_{k \geq 0} \subset(0,1)$ be real sequences satisfying the following conditions:

(i) $\lim _{k \rightarrow \infty} a_{k}=1$ (monotonically); 
(ii) $\lim _{k, r \rightarrow \infty} \frac{a_{k}-a_{r}}{1-a_{k}}=0, \forall 0<r \leq k$;

(iii) $\lim _{n \rightarrow \infty} \alpha_{n}=0$;

(iv) $\sum_{n \geq 0} \alpha_{n}=\infty$.

For an arbitrary but fixed $w \in C$, and for each $k \geq 0$, define $T_{k}: C \rightarrow C$ by

$$
T_{k} x:=\left(1-a_{k}\right) w+a_{k} T x, \quad \forall x \in C .
$$

Then the double sequence $\left\{x_{k, n}\right\}_{k \geq 0, n \geq 0}$ generated from an arbitrary $x_{0,0} \in C$ by

$$
x_{k, n+1}=\left(1-\alpha_{n}\right) x_{k, n}+\alpha_{n} T_{k} x_{k, n}, \quad k, n \geq 0,
$$

converges strongly to a fixed point $x_{\infty}^{*}$ of $T$ in $C$.

The two most popular iteration procedures for obtaining fixed points of $T$, when the Banach principle fails, are doubly Mann iterations with errors [29] defined by

$$
u_{k, n+1}=\left(1-\alpha_{n}\right) u_{k, n}+\alpha_{n} T u_{k, n}+\alpha_{n} u_{n}
$$

and doubly Ishikawa iterations with errors defined by

$$
\begin{aligned}
& x_{k, n+1}=\left(1-\alpha_{n}\right) x_{k, n}+\alpha_{n} T z_{k, n}+\alpha_{n} v_{n}, \\
& z_{k, n}=\left(1-\beta_{n}\right) x_{k, n}+\beta_{n} T x_{k, n}+\beta_{n} w_{n} .
\end{aligned}
$$

The sequences $\left\{\alpha_{n}\right\} \subset(0,1),\left\{\beta_{n}\right\} \subset[0,1)$ satisfy

$$
\lim _{n \rightarrow \infty} \alpha_{n}=\lim _{n \rightarrow \infty} \beta_{n}=0, \quad \sum_{n=1}^{\infty} \alpha_{n}=\infty .
$$

A reasonable conjecture is that the doubly Ishikawa iteration with error and the corresponding doubly Mann iteration with error are equivalent for all maps for which either method provides convergence to a fixed point.

In the present paper, we define the following iteration which will be called the general Mann iteration process with errors:

$$
S u_{k, n+1}=\left(1-\alpha_{n}\right) S u_{k, n}+\alpha_{n} T u_{k, n}+\alpha_{n} u_{n} .
$$

Using this general Mann iteration process, we give a strong convergence theorem in the double-sequence setting.

It should be remarked that in (4), if we put $S=I$, where $I$ denotes the identity mapping, then we obtain the Mann iteration process with errors (see [30]).

The general doubly Ishikawa iteration with error is defined by

$$
\begin{aligned}
& S x_{k, n+1}=\left(1-\alpha_{n}\right) S x_{k, n}+\alpha_{n} T z_{k, n}+\alpha_{n} v_{n}, \\
& S z_{k, n}=\left(1-\beta_{n}\right) S x_{k, n}+\beta_{n} T x_{k, n}+\beta_{n} w_{n} .
\end{aligned}
$$

The sequences $\left\{\alpha_{n}\right\} \subset(0,1),\left\{\beta_{n}\right\} \subset[0,1)$ satisfy

$$
\lim _{n \rightarrow \infty} \alpha_{n}=\lim _{n \rightarrow \infty} \beta_{n}=0, \quad \sum_{n=1}^{\infty} \alpha_{n}=\infty .
$$


It should be remarked that in (5) and (6), if we put $S=I$, where $I$ denotes the identity mapping, then we obtain the Ishikawa iteration process with errors (see [31, 32]).

\section{A strong convergence theorem}

In this section, it is proved that a general Mann-type double-sequence iteration process with error converges strongly to a coincidence point of the continuous pseudo-contractive mappings $S$ and $T$ both of them map $C$ into $C$ (where $C$ is a bounded closed convex nonempty subset of a (real) Hilbert space). Now, we give the following theorem.

Theorem 2.1 Let $C$ be a bounded closed convex nonempty subset of a (real) Hilbert space $H$, and let $S, T: C \rightarrow C$ be continuous pseudo-contractive maps. Let $\left\{\alpha_{n}\right\}_{n \geq 0},\left\{a_{k}\right\}_{k \geq 0} \subset$ $(0,1)$ be real sequences satisfying the following conditions:

(i) $\lim _{k \rightarrow \infty} a_{k}=1$ (monotonically);

(ii) $\lim _{k, r \rightarrow \infty} \frac{a_{k}-a_{r}}{1-a_{k}}=0, \forall 0<r \leq k$;

(iii) $\lim _{n \rightarrow \infty} \alpha_{n}=0$;

(iv) $\sum_{n>0} \alpha_{n}=\infty$.

For an arbitrary but fixed $w \in C$, and for each $k \geq 0$, define $T_{k}: C \rightarrow C$ by

$$
T_{k} x=\left(1-a_{k}\right) w+a_{k} T x+\left(1-a_{k}\right) u_{k}, \quad \forall x \in C
$$

Then the double sequence $\left\{x_{k, n}\right\}_{k \geq 0, n \geq 0}$ generated from an arbitrary $x_{0,0} \in C$ by

$$
S x_{k, n+1}=\left(1-\alpha_{n}\right) S x_{k, n}+\alpha_{n} T_{k} x_{k, n}+\left(1-\alpha_{n}\right) u_{k, n}, \quad k, n \geq 0,
$$

converges strongly to a coincidence point $x_{\infty}^{*}$ of $S$ and $T \in C$.

Proof Clearly, $C F(T) \neq \emptyset$ and $C F(S) \neq \emptyset$ (see e.g. [33]), where the set of coincidence points of $T$ is denoted by $C F(T)$ and the set of coincidence points of $S$ is denoted by $C F(S)$.

Now, we have

$$
\left\langle T_{k} x-T_{k} y, S x-S y\right\rangle=a_{k}\langle T x-T y, S x-S y\rangle \leq a_{k}\|S x-S y\|^{2}
$$

so that for all $k \geq 0, T_{k}$ is continuous and strongly pseudo-contractive. Also, $C$ is invariant under $T_{k}$ for all $k$ by convexity. Hence, $T_{k}$ has a unique fixed point $x_{k}^{*} \in C, \forall k \geq 0$. It thus suffices to prove the following:

(1) for each fixed $k \geq 0, S x_{k, n} \rightarrow S x_{k}^{*} \in C$ as $n \rightarrow \infty$;

(2) $S x_{k}^{*} \rightarrow S x_{\infty}^{*} \in C$ as $k \rightarrow \infty$;

(3) $x_{\infty}^{*} \in C F(S) \cap C F(T)$.

The first is known, but for completeness we give the details.

Now, let $d=\operatorname{diam} C$ and $b_{k}=1-a_{k} \in(0,1), \forall k$. Then

$$
\begin{aligned}
& \left\|S x_{k, n+1}-S x_{k}^{*}\right\|^{2} \\
& \quad=\left\|\left(1-\alpha_{n}\right) S x_{k, n}+\alpha_{n} T_{k} x_{k, n}+\left(1-\alpha_{n}\right) u_{k, n}-S x_{n}^{*}\right\|^{2} \\
& \quad=\left\|S x_{k, n}-S x_{k}^{*}-\alpha_{n}\left(S x_{k, n}-T_{k} x_{k, n}\right)+\left(1-\alpha_{n}\right) u_{k, n}\right\|^{2} \\
& \quad=\left\|S x_{k, n}-S x_{k}^{*}\right\|^{2}-2 \alpha_{n}\left(S x_{k, n}-T_{k} x_{k, n}, S x_{k, n}-S x_{k}^{*}\right\rangle-2\left(1-\alpha_{n}\right)\left\langle u_{k, n}, S x_{k, n}-S x_{k}^{*}\right\rangle
\end{aligned}
$$




$$
\begin{aligned}
& +\alpha_{n}^{2}\left\|S x_{k, n}-T_{k} x_{k, n}\right\|^{2}+2 \alpha_{n}\left(1-\alpha_{n}\right)\left\langle x_{k, n}-T_{k} x_{k, n}, u_{n}\right\rangle+\left(1-\alpha_{n}\right)^{2}\left\|u_{k, n}\right\|^{2} \\
\leq & \left\|S x_{k, n}-S x_{k}^{*}\right\|^{2}-2 \alpha_{n} a_{k}\left\|S x_{k, n}-S x_{k}^{*}\right\|^{2}-2\left(1-\alpha_{n}\right)\left\|u_{k, n}\right\|\left\|S x_{k, n}-S x_{k}^{*}\right\| \alpha_{n}^{2} d^{2} \\
& +2 \alpha_{n}\left(1-\alpha_{n}\right)\left\|S x_{k, n}-T_{k} x_{k, n}\right\|\left\|u_{k, n}\right\|+\left(1-\alpha_{n}\right)^{2}\left\|u_{k, n}\right\|^{2} .
\end{aligned}
$$

If we set

$$
\theta_{k, n}=\left\|S x_{k, n}-S x_{k}^{*}\right\|, \quad \delta_{k, n}=2 a_{k} \alpha_{n},
$$

then from (5) we obtain

$$
\begin{aligned}
\theta_{k, n+1}^{2} \leq & \left(1-\delta_{k, n}\right) \theta_{k, n}^{2}+d^{2} \alpha_{n}^{2}+\left(2 \alpha_{n}\left(1-\alpha_{n}\right) d-2\left(1-\alpha_{n}\right) \theta_{k, n}\right)\left\|u_{k, n}\right\| \\
& +\left(1-\alpha_{n}\right)^{2}\left\|u_{k, n}\right\|^{2} \\
= & \left(1-\delta_{k, n}\right) \theta_{k, n}^{2}+d^{2} \alpha_{n}^{2}+\left\{\left(1-\alpha_{n}\right)\left(2 \alpha_{n} d+\left(1-\alpha_{n}\right)\left\|u_{k, n}\right\|-2 \theta_{k, n}\right)\right\}\left\|u_{k, n}\right\| .
\end{aligned}
$$

Observing that

$$
d^{2} \alpha_{n}^{2}=O\left(\delta_{k, n}\right), \quad \lim _{n \rightarrow \infty} \delta_{k, n}=0 \quad \text { and } \quad \sum_{n \geq 0} d^{2} \alpha_{n}^{2}=\infty,
$$

we obtain $\theta_{k, n} \rightarrow 0$ as $n \rightarrow \infty$. So the first part is proved. Now, we have

$$
\begin{aligned}
\left\|S x_{k}^{*}-T x_{k}^{*}\right\| & =\left\|S x_{k}^{*}-a_{k}^{-1} S x_{k}^{*}-a_{k}^{-1}\left(1-a_{k}\right) w-a_{k}^{-1}\left(1-a_{k}\right) u_{k}\right\| \\
& =\left\|\left(1-\frac{1}{a_{k}}\right) S x_{k}^{*}-\left(\frac{1-a_{k}}{a_{k}}\right)\left(w+u_{k}\right)\right\| \\
& =\left\|-\left(\frac{1-a_{k}}{a_{k}}\right) S x_{k}^{*}-\left(\frac{1-a_{k}}{a_{k}}\right)\left(w+u_{k}\right)\right\| \\
& =\left(\frac{1-a_{k}}{a_{k}}\right)\left\|-\left(S x_{k}^{*}+w+u_{k}\right)\right\| \\
& \leq\left(\frac{1-a_{k}}{a_{k}}\right)\left(\left\|S x_{k}^{*}\right\|+\|w\|+\left\|u_{k}\right\|\right) \\
& \leq\left(\frac{1-a_{k}}{a_{k}}\right)\left(2 d+\left\|u_{k}\right\|\right),
\end{aligned}
$$

which implies that

$$
\lim _{k \rightarrow \infty}\left\|S x_{k}^{*}-T x_{k}^{*}\right\| \leq 0
$$

Then

$$
\lim _{k \rightarrow \infty}\left\|S x_{k}^{*}-T x_{k}^{*}\right\|=0
$$

hence $\left\{x_{k}^{*}\right\}$ is a coincidence point sequence for $S$ and $T$. Also, assuming that $x_{\infty}^{*}$ is a coincidence point of $S$ and $T$, then

$$
\left\|S x_{\infty}^{*}-T x_{\infty}^{*}\right\| \leq \lim _{k \rightarrow \infty}\left(2 d+\left\|u_{k}\right\|\right)\left(\frac{1-a_{k}}{a_{k}}\right)=0 .
$$


Now, for all $0<r \leq k$, we have

$$
\begin{aligned}
&\left\|S x_{k}^{*}-S x_{r}^{*}\right\|^{2} \\
&=\left\langle S x_{k}^{*}-S x_{r}^{*}, S x_{k}^{*}-S x_{r}^{*}\right\rangle=\left\langle T_{k} x_{k}^{*}-T_{r} x_{r}^{*}, S x_{k}^{*}-S x_{r}^{*}\right\rangle \\
&=\left\langle 7 w+a_{k} T x_{k}-a_{r} T x_{r}+\left(1-a_{k}\right) u_{k}-\left(1-a_{r}\right) u_{r}, S x_{k}^{*}-S x_{r}^{*}\right\rangle \\
&=\left(a_{r}-a_{k}\right)\left\langle w, S x_{k}^{*}-S x_{r}^{*}\right\rangle+\left\langle a_{k} T x_{k}^{*}-a_{r} T x_{r}^{*}, S x_{k}^{*}-S x_{r}^{*}\right\rangle \\
& \quad+\left(1-a_{k}\right)\left\langle u_{k}, S x_{k}^{*}-S x_{r}^{*}\right\rangle-\left(1-a_{r}\right)\left\langle u_{r}, S x_{k}^{*}-S x_{r}^{*}\right\rangle \\
&=\left(a_{r}-a_{k}\right)\left\langle w, S x_{k}^{*}-S x_{r}^{*}\right\rangle+\left(a_{k}-a_{r}\right)\left\langle T x_{r}^{*}, S x_{k}^{*}-S x_{r}^{*}\right\rangle+a_{k}\left\langle T x_{k}^{*}-T x_{r}^{*}, S x_{k}^{*}-S x_{r}^{*}\right\rangle \\
& \quad+\left(1-a_{k}\right)\left\langle u_{k}, S x_{k}^{*}-S x_{r}^{*}\right\rangle-\left(1-a_{r}\right)\left\langle u_{r}, S x_{k}^{*}-S x_{r}^{*}\right\rangle\left\|S x_{k}^{*}-S x_{r}^{*}\right\|^{2} \\
& \leq\left(a_{k}-a_{r}\right)\|w\|\left\|S x_{k}^{*}-S x_{r}^{*}\right\|+\left(a_{k}-a_{r}\right)\left\|T x_{r}^{*}\right\|\left\|S x_{k}^{*}-S x_{r}^{*}\right\|+a_{k}\left\|S x_{k}^{*}-S x_{r}^{*}\right\|^{2} \\
& \quad+\left(1-a_{k}\right)\left\|u_{k}\right\|\left\|S x_{k}^{*}-S x_{r}^{*}\right\|-\left(1-a_{r}\right)\left\|u_{r}\right\|\left\|S x_{k}^{*}-S x_{r}^{*}\right\| \\
& \leq\left(a_{k}-a_{r}\right)\left\|S x_{k}^{*}-S x_{r}^{*}\right\|\left(\|w\|+\left\|T x_{r}^{*}\right\|\right)+a_{k}\left\|S x_{k}^{*}-S x_{r}^{*}\right\|^{2} \\
&+\left(\left(1-a_{k}\right)\left\|u_{k}\right\|-\left(1-a_{r}\right)\left\|u_{r}\right\|\right)\left\|S x_{k}^{*}-S x_{r}^{*}\right\| .
\end{aligned}
$$

Then we obtain

$$
\begin{aligned}
\left(1-a_{k}\right)\left\|S x_{k}^{*}-S x_{r}^{*}\right\|^{2} \leq & \left(a_{k}-a_{r}\right)\left(\|w\|+\left\|T x_{r}^{*}\right\|\right)\left\|S x_{k}^{*}-S x_{r}^{*}\right\| \\
& +\left(\left(1-a_{k}\right)\left\|u_{k}\right\|-\left(1-a_{r}\right)\left\|u_{r}\right\|\right)\left\|S x_{k}^{*}-S x_{r}^{*}\right\| .
\end{aligned}
$$

Then

$$
\begin{aligned}
\left\|S x_{k}^{*}-S x_{r}^{*}\right\|^{2} \leq & \frac{a_{k}-a_{r}}{1-a_{k}}\left(\|w\|+\left\|T x_{r}^{*}\right\|\right)\left\|S x_{k}^{*}-S x_{r}^{*}\right\| \\
& +\left(\frac{1-a_{k}}{1-a_{k}}\left\|u_{k}\right\|-\frac{1-a_{r}}{1-a_{k}}\left\|u_{r}\right\|\right)\left\|S x_{k}^{*}-S x_{r}^{*}\right\| \\
\leq & \frac{a_{k}-a_{r}}{1-a_{k}}(2 d)\left\|S x_{k}^{*}-S x_{r}^{*}\right\|+\left(\left\|u_{k}\right\|-\frac{1-a_{r}}{1-a_{k}}\left\|u_{r}\right\|\right)\left\|S x_{k}^{*}-S x_{r}^{*}\right\|,
\end{aligned}
$$

which implies that

$$
\left\|S x_{k}^{*}-S x_{r}^{*}\right\| \leq \frac{a_{k}-a_{r}}{1-a_{k}}(2 d)+\left\|u_{k}\right\|-\frac{1-a_{r}}{1-a_{k}}\left\|u_{r}\right\| .
$$

Hence,

$$
\lim _{k, r \rightarrow \infty}\left\|S x_{k}^{*}-S x_{r}^{*}\right\| \leq 2 d \lim _{k, r \rightarrow \infty}\left(\frac{a_{k}-a_{r}}{1-a_{k}}\right)+\lim _{k \rightarrow \infty}\left\|u_{k}\right\|-\lim _{k, r \rightarrow \infty}\left(\frac{1-a_{r}}{1-a_{k}} \cdot\left\|u_{r}\right\|\right)=0 .
$$

Thus $\left\{S x_{k}^{*}\right\}$ is a Cauchy sequence, and hence there exists $\left\{S x_{\infty}^{*}\right\} \in C$ such that $S x_{k}^{*} \rightarrow S x_{\infty}^{*}$ as $k \rightarrow \infty$. Therefore, the second part is proved. By continuity, $T x_{k}^{*} \rightarrow T x_{\infty}^{*}$ as $k \rightarrow \infty$. But $S x_{k}^{*}-T x_{k}^{*} \rightarrow 0$ as $k \rightarrow \infty$. Hence, $x_{\infty}^{*} \in C F(S) \cap C F(T)$. This completes the proof.

Corollary 2.1 Let $C$ be a bounded closed convex nonempty subset of a Hilbert space $H$ with $0 \in C$. Let $S, T,\left\{a_{k}\right\},\left\{\alpha_{n}\right\},\left\{x_{k, n}\right\}$ be as in Theorem 2.1 and $\forall k \geq 0$ define $T_{k}=a_{k} T+$ 
$\left(1-a_{k}\right) S u_{k}$. Then $T_{k}$ maps $C$ into itself and $\left\{x_{k, n}\right\}$ converges strongly to a coincidence point of $S$ and $T$.

Proof The proof follows from Theorem 2.1 by setting $w=0 \in C$.

Corollary 2.2 In Theorem 2.1, let $S, T$ be two nonexpansive self-mappings. Then the same conclusion is obtained.

Proof The proof of this corollary can be followed directly by observing that every nonexpansive mapping is a continuous pseudo-contraction.

Remark 2.1 If we put $u_{k}=0$ in Theorem 2.1, we obtain the result of Moore in [24].

\section{The equivalence between $S, T$-stabilities}

In this section, we give the concept of $S, T$-stabilities, then we show that $S, T$-stabilities of general doubly Mann and general doubly Ishikawa iterations are equivalent.

Let $\left\{S x_{k, n}\right\}$ be the doubly general Ishikawa iteration with errors and $\left\{S u_{k, n}\right\}$ be the general doubly Mann iteration with errors. Let $\left\{q_{k, n}\right\},\left\{p_{k, n}\right\} \subset E$ be such that $q_{0,0}=p_{0,0}$, and let $\left(\alpha_{n}\right)_{n} \subset(0,1),\left(\beta_{n}\right)_{n} \subset[0,1) ; n \in \mathbb{N}$ satisfy $(7)$ and

$$
S y_{k, n}=\left(1-\beta_{n}\right) S q_{k, n}+\beta_{n} T q_{k, n} .
$$

We consider the following nonnegative sequences for all $n \in \mathbb{N}$ :

$$
\epsilon_{k, n}:=\left\|S q_{k, n+1}-\left(1-\alpha_{n}\right) S q_{k, n}-\alpha_{n} T y_{k, n}+\alpha_{n} v_{n}\right\|
$$

and

$$
\delta_{k, n}:=\left\|S p_{k, n+1}-\left(1-\alpha_{n}\right) S p_{k, n}-\alpha_{n} T p_{k, n}+\alpha_{n} v_{n}\right\| .
$$

Let $E$ be a normed space and $T$ be a self-map of $E$. Let $x_{0,0}$ be a point of $E$, and assume that $x_{k, n+1}=f\left(T, x_{k, n}\right)$ is an iteration procedure, involving $T$, which yields a sequence $\left\{x_{k, n}\right\}$ of points from $E$. Suppose that $x_{k, n}$ converges to a fixed point $x^{*}$ of $T$. Let $\xi_{k, n}$ be an arbitrary sequence in $E$, and set

$$
\epsilon_{n}=\left\|\xi_{k, n+1}-f\left(T, \xi_{k, n}\right)\right\|, \quad \forall n \in \mathbb{N} .
$$

Definition 3.1 If $\lim _{n \rightarrow \infty} \epsilon=0 \Rightarrow \lim _{n \rightarrow \infty} \xi_{k, n}=p$, then the iteration procedure $x_{k, n+1}=$ $f\left(T, x_{k, n}\right)$ is said to be $T$-stable with respect to $T$.

Remark 3.1 In practice, such a sequence $\left\{\xi_{k, n}\right\}$ could arise in the following way. Let $x_{0,0}$ be a point in $E$. Set $x_{k, n+1}=f\left(T, x_{k, n}\right)$. Let $\xi_{0,0}=x_{0,0}$. Now $x_{0,1}=f\left(T, x_{0,0}\right)$. Because of rounding in the function $T$, a new value $\xi_{0,1}$ approximately equal to $x_{0,1}$ might be computed to yield $\xi_{1,2}$, an approximation of $f\left(T, \xi_{0,1}\right)$. This computation is continued to obtain $\left\{\xi_{k, n}\right\}$ an approximate sequence of $\left\{x_{k, n}\right\}$.

Definition 3.2 Let $E$ be a normed space and $S, T: E \rightarrow E$. 
(i) If $\lim _{k, n \rightarrow \infty} \epsilon_{k, n}=0$ implies that $\lim _{k, n \rightarrow \infty} S q_{k, n}=S x^{*}$, then the general Ishikawa iteration as defined in (5) and (6) is said to be $S, T$-stable.

(ii) If $\lim _{k, n \rightarrow \infty} \delta_{k, n}=0$ implies that $\lim _{k, n \rightarrow \infty} S p_{k, n}=S x^{*}$, then the general Mann iteration process as defined in (4) is said to be $S, T$-stable.

Remark 3.2 Let $E$ be a normed space and $S, T: E \rightarrow E$. The following are equivalent:

(a) for all $\left\{\alpha_{n}\right\} \subset(0,1),\left\{\beta_{n}\right\} \subset[0,1)$ satisfying (7), the Ishikawa iteration is $S, T$-stable,

(b) for all $\left\{\alpha_{n}\right\} \subset(0,1),\left\{\beta_{n}\right\} \subset[0,1)$ satisfying (7), $\forall\left\{q_{k, n}\right\} \subset E$,

$$
\begin{aligned}
& \lim _{k, n \rightarrow \infty} \epsilon_{k, n}=\lim _{k, n \rightarrow \infty}\left\|S q_{k, n+1}-\left(1-\alpha_{n}\right) S q_{k, n}-\alpha_{n} T y_{k, n}+\alpha_{n} v_{n}\right\|=0 \\
& \Rightarrow \quad \lim _{k, n \rightarrow \infty} S q_{k, n}=S x^{*} .
\end{aligned}
$$

Remark 3.3 Let $E$ be a normed space and $S, T: E \rightarrow E$. Then the following are equivalent:

$\left(\mathrm{a}_{1}\right)$ for all $\left\{\alpha_{n}\right\} \subset(0,1)$ satisfying (7), the general Mann iteration is $S, T$-stable,

$\left(\mathrm{a}_{2}\right)$ for all $\left\{\alpha_{n}\right\} \subset(0,1)$ satisfying $(7), \forall\left\{p_{k, n}\right\} \subset E$,

$$
\begin{aligned}
& \lim _{k, n \rightarrow \infty} \delta_{k, n}=\lim _{k, n \rightarrow \infty}\left\|S p_{k, n+1}-\left(1-\alpha_{n}\right) S p_{k, n}-\alpha_{n} T p_{k, n}+\alpha_{n} v_{n}\right\|=0 \\
& \Rightarrow \quad \lim _{k, n \rightarrow \infty} S p_{k, n}=S x^{*} .
\end{aligned}
$$

The next result states that these two methods of iterations with errors are equivalent from the $S, T$-stability point of view under certain restrictions.

Theorem 3.1 Let E be a normed space and $S, T: E \rightarrow$ E. Then the following are equivalent:

(I) For all $\left\{\alpha_{n}\right\} \subset(0,1),\left\{\beta_{n}\right\} \subset[0,1)$ satisfying (7), the general Ishikawa iteration process as defined by (5) and (6) is $S, T$-stable.

(II) For all $\left\{\alpha_{n}\right\} \subset(0,1)$, satisfying (7), the general Mann iteration process as defined in (4) is $S, T$-stable.

Proof Let

$$
M:=\max \left\{\sup _{k, n \in \mathbb{N}}\left\{\left\|T\left(y_{k, n}\right)\right\|\right\}, \sup _{k, n \in \mathbb{N}}\left\{\left\|T\left(q_{k, n}\right)\right\|\right\}, \sup _{k, n \in N}\left\{\left\|T\left(p_{k, n}\right)\right\|\right\}, \sup _{n \in \mathbb{N}}\left\{\left\|u_{n}\right\|\right\}\right\} .
$$

Since the general Mann and general Ishikawa iterations converge and $M<\infty$, Remarks 3.1 and 3.2 assure that (I) $\Leftrightarrow$ (II) is equivalent to $(\mathrm{b}) \Leftrightarrow\left(\mathrm{a}_{2}\right)$. We shall prove that $(\mathrm{b}) \Rightarrow\left(\mathrm{a}_{2}\right)$. In (b) and (13) set $S q_{k, n}:=S p_{k, n}$, we obtain

$$
\begin{aligned}
& \left\|S p_{k, n+1}-\left(1-\alpha_{n}\right) S p_{k, n}-\alpha_{n} T p_{k, n}+\alpha_{n} u_{n}\right\| \\
& \quad \leq\left\|S p_{k, n+1}-\left(1-\alpha_{n}\right) S p_{k, n}-\alpha_{n} T y_{k, n}\right\|+\left\|\alpha_{n} T y_{k, n}-\alpha_{n} T p_{n}+\alpha_{n} u_{n}\right\| \\
& \quad \leq\left\|S p_{k, n+1}-\left(1-\alpha_{n}\right) S p_{k, n}-\alpha_{n} T y_{k, n}\right\|+\alpha_{n}\left(\left\|T y_{k, n}\right\|+\left\|T p_{k, n}\right\|+\left\|u_{n}\right\|\right) \\
& \quad \leq\left\|S p_{k, n+1}-\left(1-\alpha_{n}\right) S p_{k, n}-\alpha_{n} T y_{k, n}\right\|+3 \alpha_{n} M \rightarrow 0 \quad \text { as } n \rightarrow \infty .
\end{aligned}
$$

Condition (b) assures that

$$
\lim _{k, n \rightarrow \infty}\left\|S p_{k, n+1}-\left(1-\alpha_{n}\right) S p_{k, n}-\alpha_{n} T y_{k, n}+\alpha_{n} u_{n}\right\|=0 \Rightarrow \lim _{k, n \rightarrow \infty} S p_{k, n}=S x^{*}
$$


Thus, for $\left\{S p_{k, n}\right\}$ satisfying

$$
\lim _{k, n \rightarrow \infty}\left\|S p_{k, n+1}-\left(1-\alpha_{n}\right) S p_{k, n}-\alpha_{n} T y_{k, n}+\alpha_{n} u_{n}\right\|=0
$$

we have shown that

$$
\lim _{k, n \rightarrow \infty} S p_{k, n}=S x^{*}
$$

Conversely, we prove $\left(\mathrm{a}_{2}\right) \Rightarrow(\mathrm{b})$. In $\left(\mathrm{a}_{2}\right)$ and (14) set $S p_{k, n}=S q_{k, n}$ to obtain

$$
\begin{aligned}
& \left\|S q_{k, n+1}-\left(1-\alpha_{n}\right) S q_{k, n}-\alpha_{n} T y_{k, n}+\alpha_{n} u_{n}\right\| \\
& \quad \leq\left\|S q_{k, n+1}-\left(1-\alpha_{n}\right) S q_{k, n}-\alpha_{n} T s_{k, n}\right\|+\left\|\alpha_{n} T y_{k, n}-\alpha_{n} T s_{n}+\alpha_{n} u_{n}\right\| \\
& \quad \leq\left\|S q_{k, n+1}-\left(1-\alpha_{n}\right) S q_{k, n}-\alpha_{n} T S q_{k, n}\right\|+3 \alpha_{n} M \rightarrow 0 \text { as } n \rightarrow \infty .
\end{aligned}
$$

Condition $\left(\mathrm{a}_{2}\right)$ assures that

$$
\lim _{k, n \rightarrow \infty}\left\|S q_{k, n+1}-\left(1-\alpha_{n}\right) S q_{k, n}-\alpha_{n} T S q_{k, n}+\alpha_{n} u_{n}\right\|=0 \Rightarrow \lim _{k, n \rightarrow \infty} S q_{k, n}=S x^{*}
$$

Thus, for $\left\{S q_{k, n}\right\}$ satisfying

$$
\lim _{k, n \rightarrow \infty}\left\|S q_{k, n+1}-\left(1-\alpha_{n}\right) S q_{k, n}-\alpha_{n} T y_{k, n}+\alpha_{n} u_{n}\right\|=0
$$

we have shown that

$$
\lim _{k, n \rightarrow \infty} S q_{k, n}=S x^{*}
$$

This completes the proof of the theorem.

Corollary 3.1 Let E be a normed space and $S, T: E \rightarrow E$. Then the following are equivalent:

(i) For all $\left\{\alpha_{n}\right\} \subset(0,1),\left\{\beta_{n}\right\} \subset[0,1)$ satisfying (7), the Ishikawa iteration process defined by

$$
\begin{aligned}
& x_{k, n+1}=\left(1-\alpha_{n}\right) x_{k, n}+\alpha_{n} T z_{k, n}+\alpha_{n} v_{n}, \\
& z_{k, n}=\left(1-\beta_{n}\right) x_{k, n}+\beta_{n} T x_{k, n}+\beta_{n} w_{n}
\end{aligned}
$$

is T-stable.

(ii) For all $\left\{\alpha_{n}\right\} \subset(0,1)$, satisfying (7), the Mann iteration process defined by

$$
u_{k, n+1}=\left(1-\alpha_{n}\right) u_{k, n}+\alpha_{n} T u_{k, n}+\alpha_{n} u_{n}
$$

is T-stable.

Proof The proof of this result can be obtained directly by setting $S=I$ in Theorem 3.1, where $I$ denotes the identity mapping. 


\section{Application}

In this section, we investigate the solvability of certain nonlinear functional equations in a Banach space $X$ by the help of compatible mappings of type (B) in the double-sequence setting.

The concept of compatible mappings of type (B) was introduced by Pathak and Khan (see [34]).

Definition 4.1 (see [34] and [29]) Let $S$ and $T$ be mappings from a normed space $E$ into itself. The mappings $S$ and $T$ are said to be compatible mappings of type (B) if

$$
\lim _{n \rightarrow \infty}\left\|S T x_{n}-T T x_{n}\right\| \leq \frac{1}{2}\left[\lim _{n \rightarrow \infty}\left\|S T x_{n}-S t\right\|+\lim _{n \rightarrow \infty}\left\|S t-S S x_{n}\right\|\right]
$$

and

$$
\lim _{n \rightarrow \infty}\left\|T S x_{n}-S S x_{n}\right\| \leq \frac{1}{2}\left[\lim _{n \rightarrow \infty}\left\|T S x_{n}-T t\right\|+\lim _{n \rightarrow \infty}\left\|T t-T T x_{n}\right\|\right]
$$

whenever $\left\{x_{n}\right\}$ is a sequence in $E$ such that $\lim _{n \rightarrow \infty} S x_{n}=\lim _{n \rightarrow \infty} T x_{n}=t$ for some $t \in E$.

Now, we extend the above definition to double-sequence setting as follows.

Definition 4.2 Let $S$ and $T$ be mappings from a normed space $E$ into itself. The mappings $S$ and $T$ are said to be compatible mappings of type (B) if

$$
\lim _{n, m \rightarrow \infty}\left\|S T x_{n, m}-T T x_{n, m}\right\| \leq \frac{1}{2}\left[\lim _{n, m \rightarrow \infty}\left\|S T x_{n, m}-S t\right\|+\lim _{n, m \rightarrow \infty}\left\|S t-S S x_{n, m}\right\|\right]
$$

and

$$
\lim _{n, m \rightarrow \infty}\left\|T S x_{n, m}-S S x_{n, m}\right\| \leq \frac{1}{2}\left[\lim _{n, m \rightarrow \infty}\left\|T S x_{n, m}-T t\right\|+\lim _{n, m \rightarrow \infty}\left\|T t-T T x_{n, m}\right\|\right]
$$

whenever $\left\{x_{n, m}\right\}$ is a sequence in $E$ such that $\lim _{n, m \rightarrow \infty} S x_{n, m}=\lim _{n, m \rightarrow \infty} T x_{n, m}=t$ for some $t \in E$.

Now, we state and prove the following result.

Theorem 4.1 Let $\left\{f_{n, m}\right\},\left\{g_{n, m}\right\},\left\{t_{n, m}\right\}$ and $\left\{r_{n, m}\right\}$ be sequences of elements in a Banach space X. Let $\left\{v_{n, m}\right\}$ be the unique solution of the system of equations

$$
\left\{\begin{array}{l}
F v-A B v=f_{n, m} \\
F v-B B v=g_{n, m} \\
F v-S T v=t_{n, m} \\
F v-T T v=r_{n, m}
\end{array}\right.
$$

where $F, A, B, S, T: X \rightarrow X$ satisfy the following conditions:

$\left(\mathrm{d}_{1}\right)$ The pairs $\{A, S\}$ and $\{B, T\}$ are compatible of type $(B)$,

$\left(\mathrm{d}_{2}\right) A^{2}=B^{2}=S^{2}=T^{2}=I$, where $I$ denotes the identity mapping, and 
$\left(\mathrm{d}_{3}\right)$

$$
\begin{array}{r}
\|A x-B y\|^{2} \leq q \max \left\{\|S x-T y\|^{2},\|S x-A x\|^{2},\|S x-A x\| \times\|T y-B y\|,\right. \\
\left.\|T y-A x\| \times\|S x-B y\|, \frac{1}{2}\left[\|T y-A x\|^{2}+\|S x-B y\|^{2}\right]\right\}
\end{array}
$$

for all $x, y \in X$, where $q \in(0,1)$. If $F v=v$ and

$$
\lim _{n, m \rightarrow \infty}\left\|f_{n, m}\right\|=\lim _{n, m \rightarrow \infty}\left\|g_{n, m}\right\|=\lim _{n, m \rightarrow \infty}\left\|t_{n, m}\right\|=\lim _{n, m \rightarrow \infty}\left\|r_{n, m}\right\|=0
$$

then the sequence $\left\{v_{n, m}\right\}$ converges to the solution of the equation

$$
v=F v=A v=B v=S v=T v .
$$

Proof We will show that $\left\{v_{n, m}\right\}$ is a Cauchy sequence. Since

$$
\begin{aligned}
& \left\|v_{n, m}-v_{n_{1}, m_{1}}\right\|^{2} \\
& =\left[\left\|v_{n, m}-S T v_{n, m}\right\|+\left\|S T v_{n, m}-T T v_{n, m}\right\|+\left\|T T v_{n, m}-A B v_{n, m}\right\|\right. \\
& \left.+\left\|A B v_{n, m}-B B v_{n_{1}, m_{1}}\right\|+\left\|B B v_{n_{1}, m_{1}}-v_{n_{1}, m_{1}}\right\|\right]^{2} \\
& \leq\left[\left\|v_{n, m}-S T v_{n, m}\right\|+\left\|S T v_{n, m}-T T v_{n, m}\right\|+\left\|T T v_{n, m}-A B v_{n, m}\right\|\right. \\
& \left.+\left\|B B v_{n_{1}, m_{1}}-v_{n_{1}, m_{1}}\right\|\right]^{2}+2\left[\left\|v_{n, m}-S T v_{n, m}\right\|+\left\|S T v_{n, m}-T T v_{n, m}\right\|\right. \\
& \left.+\left\|T T v_{n, m}-A B v_{n, m}\right\|+\left\|B B v_{n_{1}, m_{1}}-v_{n_{1}, m_{1}}\right\|\right]\left[\left\|A B v_{n, m}-v_{n, m}\right\|\right. \\
& \left.+\left\|v_{n, m}-v_{n_{1}, m_{1}}\right\|+\left\|v_{n_{1}, m_{1}}-B B v_{n_{1}, m_{1}}\right\|\right]+\left\|A B v_{n, m}-B B v_{n_{1}, m_{1}}\right\|^{2} \\
& \leq\left[\left\|v_{n, m}-S T v_{n, m}\right\|+\left\|S T v_{n, m}-T T v_{n, m}\right\|+\left\|T T v_{n, m}-A B v_{n, m}\right\|\right. \\
& \left.+\left\|B B v_{n_{1}, m_{1}}-v_{n_{1}, m_{1}}\right\|\right]^{2}+2\left[\left\|v_{n, m}-S T v_{n, m}\right\|+\left\|S T v_{n, m}-T T v_{n, m}\right\|\right. \\
& \left.+\left\|T T v_{n, m}-A B v_{n, m}\right\|+\left\|B B v_{n_{1}, m_{1}}-v_{n_{1}, m_{1}}\right\|\right]\left[\left\|A B v_{n, m}-v_{n, m}\right\|\right. \\
& \left.+\left\|v_{n, m}-v_{n_{1}, m_{1}}\right\|+\left\|v_{n_{1}, m_{1}}-B B v_{n_{1}, m_{1}}\right\|\right]+q \max \left\{\left\|S B v_{n, m}-T B v_{n_{1}, m_{1}}\right\|^{2},\right. \\
& \left\|S B v_{n, m}-A B v_{n, m}\right\|^{2},\left\|S B v_{n, m}-A B v_{n, m}\right\| \times\left\|T B v_{n_{1}, m_{1}}-B B v_{n_{1}, m_{1}}\right\|, \\
& \left\|T B v_{n_{1}, m_{1}}-A B v_{n, m}\right\| \times\left\|S B v_{n, m}-B B v_{n_{1}, m_{1}}\right\|, \frac{1}{2}\left[\left\|T B v_{n_{1}, m_{1}}-A B v_{n, m}\right\|^{2}\right. \\
& \left.\left.+\left\|S B v_{n, m}-B B v_{n_{1}, m_{1}}\right\|^{2}\right]\right\}
\end{aligned}
$$

Letting $n, n_{1} \rightarrow \infty$ with $m>n$ and $m_{1}>n_{1}$, we deduce

$$
\lim _{n, n_{1} \rightarrow \infty}\left\|v_{n, m}-v_{n_{1}, m_{1}}\right\|^{2} \leq q \lim _{n, n_{1} \rightarrow \infty}\left\|v_{n, m}-v_{n_{1}, m_{1}}\right\|^{2},
$$

which implies that

$$
\lim _{n, n_{1} \rightarrow \infty}\left\|v_{n, m}-v_{n_{1}, m_{1}}\right\|^{2}=0
$$


Thus $\left\{v_{n, m}\right\}$ is a Cauchy sequence and converges to a point $v$ in $X$. Further,

$$
\begin{aligned}
\|v-A B v\| \leq & \left\|v-v_{n, m}\right\|+\left\|v_{n}-B B v_{n, m}\right\|+\left\|B B v_{n, m}-A B v\right\| \\
\leq & \left\|v-v_{n, m}\right\|+\left\|v_{n, m}-B B v_{n, m}\right\|+q \max \left\{\left\|S B v_{n, m}-T B v\right\|^{2},\right. \\
& \|S B v-A B v\|^{2},\|S B v-A B v\| \times\left\|T B v_{n, m}-B B v_{n, m}\right\|,\left\|T B v_{n, m}-A B v\right\| \\
& \left.\times\left\|S B v-B B v_{n, m}\right\|, \frac{1}{2}\left\|T B v_{n}-A B v\right\|^{2}+\left\|S B v-B B v_{n}\right\|^{2}\right\}^{\frac{1}{2}} \\
\leq & \left\|v-v_{n, m}\right\|+\left\|v_{n, m}-B B v_{n, m}\right\|+q \max \left\{\left[\left\|S B v_{n, m}-v_{n, m}\right\|+\left\|v_{n, m}-v\right\|\right]^{2}\right. \\
& \times[\|S B v-v\|+\|v-A B v\|]^{2},\|S B v-A B v\| \times\left\|T B v_{n}-B B v_{n, m}\right\|, \\
& {\left[\left\|T B v_{n, m}-v_{n, m}\right\|+\left\|v_{n, m}-A B v\right\|\right] \times\left[\|S B v-v\|+\left\|v-B B v_{n, m}\right\|\right], } \\
& \left.\frac{1}{2}\left[\left\|T B v_{n, m}-A B v\right\|^{2}+\left\|S B v-B B v_{n, m}\right\|^{2}\right]\right\}^{\frac{1}{2}} \cdot
\end{aligned}
$$

Letting $n \rightarrow \infty$, we get $v=A B v$, which from $\left(\mathrm{d}_{2}\right)$ implies that $A v=T \nu$. Similarly, $T v=S v$.

From $\left(\mathrm{d}_{1}\right)$, we now have

$$
A B v=B A v=v=S B v=B S v=T B v=B T v
$$

Using (i) and $\left(\mathrm{d}_{2}\right)$, we have

$$
\begin{aligned}
\|v-B v\|^{2}= & \left\|A^{2} v-B v\right\|^{2} \\
\leq & q \max \left\{\|S A v-T v\|^{2},\left\|S A v-A^{2} v\right\|^{2},\left\|S A v-A^{2} v\right\| \times\|T v-B v\|,\right. \\
& \left.\left\|T v-A^{2} v\right\| \times\|S A v-B v\|, \frac{1}{2}\left[\left\|T v-A^{2} v\right\|^{2}+\|S A v-B v\|^{2}\right]\right\} \\
\leq & q \max \left\{\|v-T v\|^{2}, 0,0,\|T v-v\| \times\|v-B v\|, \frac{1}{2}\|T v-v\|^{2}+\|v-B v\|\right\} \\
\leq & q \max \left\{\|v-T v\|^{2}, 0,0,\|v-T v\|^{2},\|T v-v\|^{2}\right\},
\end{aligned}
$$

which implies that $v=T v$. It follows that

$$
T v=T S v=S T v=v=A B v=B A v=B T v=T B v
$$

completing the proof of the theorem.

As a consequence of Theorem 4.1, we have the following corollary.

Corollary 4.1 Let $\left\{f_{n, m}\right\},\left\{g_{n, m}\right\},\left\{t_{n, m}\right\}$ and $\left\{r_{n, m}\right\}$ be sequences of elements in a Banach space $X$. Let $\left\{v_{n, m}\right\}$ be the unique solution of the system of equations

$$
\left\{\begin{array}{l}
v-A B v=f_{n, m}, \\
v-B B v=g_{n, m}, \\
v-S T v=t_{n, m}, \\
v-T T v=r_{n, m},
\end{array}\right.
$$


where $A, B, S, T: X \rightarrow X$ satisfy the following conditions:

$\left(\mathrm{d}_{1}\right)$ The pairs $\{A, S\}$ and $\{B, T\}$ are compatible of type $(B)$,

$\left(\mathrm{d}_{2}\right) A^{2}=B^{2}=S^{2}=T^{2}=I$, where I denotes the identity mapping, and

$\left(\mathrm{d}_{3}\right)$

$$
\begin{array}{r}
\|A x-B y\|^{2} \leq q \max \left\{\|S x-T y\|^{2},\|S x-A x\|^{2},\|S x-A x\| \times\|T y-B y\|,\right. \\
\left.\|T y-A x\| \times\|S x-B y\|, \frac{1}{2}\left[\|T y-A x\|^{2}+\|S x-B y\|^{2}\right]\right\}
\end{array}
$$

for all $x, y \in X$, where $q \in(0,1)$. If

$$
\lim _{n, m \rightarrow \infty}\left\|f_{n, m}\right\|=\lim _{n, m \rightarrow \infty}\left\|g_{n, m}\right\|=\lim _{n, m \rightarrow \infty}\left\|t_{n, m}\right\|=\lim _{n, m \rightarrow \infty}\left\|r_{n, m}\right\|=0,
$$

then the sequence $\left\{v_{n, m}\right\}$ converges to the solution of the equation

$$
v=A v=B v=S v=T v .
$$

Proof The proof can be obtained by putting $F=I$ in Theorem 4.1, where $I$ denotes the identity mapping.

Open problem It is still an open problem to extend some defined iterative schemes in the sense of double-sequence setting. For some recent studies on various iterative schemes, we refer to $[1,35-39]$ and others.

\section{Competing interests}

The authors declare that they have no competing interests.

\section{Authors' contributions}

All authors contributed equally to the writing of this paper. All authors read and approved the final manuscript.

\section{Author details}

${ }^{1}$ Department of Mathematics, Faculty of Science, Sohag University, Sohag, 82524, Egypt. ${ }^{2}$ Mathematics Department, Faculty of Science, Taif University, El-Hawiyah, P.O. Box 888, El-Taif, 5700, Saudi Arabia. ${ }^{3}$ Department of Mathematics, Faculty of Science, Assiut University, Assiut, Egypt. ${ }^{4}$ Department of Mathematics, University College, Umm Al-Qura University, Mecca, Saudi Arabia.

\section{Acknowledgements}

The authors would like to thank Scientific Research Deanship at Umm Al-Qura University (Project ID 43305020) for the financial support.

\section{Received: 9 February 2014 Accepted: 15 April 2014 Published: 16 May 2014}

\section{References}

1. Berinde, V: Iterative Approximation of Fixed Points, 2nd edn. Lecture Notes in Mathematics, vol. 1912. Springer, Berlin (2007)

2. Cirić, L, Rafiq, A, Radenović, S, Rajović, M, Ume, JS: On Mann implicit iterations for strongly accretive and strongly pseudo-contractive mappings. Appl. Math. Comput. 198, 128-137 (2008)

3. Mann, WR: Mean value methods in iteration. Proc. Am. Math. Soc. 4, 506-510 (1953)

4. Browder, FE, Petryshyn, WV: Construction of fixed points of nonlinear mappings in Hilbert spaces. J. Math. Anal. Appl. 20, 197-228 (1967)

5. Chidume, CE: Approximation of fixed points of strongly pseudocontractive mappings. Proc. Am. Math. Soc. 120(2), 545-551 (1994)

6. Huang, Z, Fanwei, B: The equivalence between the convergence of Ishikawa and Mann iterations with errors for strongly successively pseudocontractive mappings without Lipschitzian assumption. J. Math. Anal. Appl. 325(1), 586-594 (2007) 
7. Kim, $\mathrm{TH}, \mathrm{Xu}, \mathrm{HK}$ : Strong convergence of modified Mann iterations for asymptotically nonexpansive mappings and semigroups. Nonlinear Anal., Theory Methods Appl. 64(5), 1140-1152 (2006)

8. Özdemir, M, Akbulut, S: On the equivalence of some fixed point iterations. Kyungpook Math. J. 46, 211-217 (2006)

9. Park, JA: Mann-iteration process for the fixed point of strictly pseudocontractive mapping in some Banach spaces. J. Korean Math. Soc. 31(3), 333-337 (1994)

10. Park, JY, Jeong, JUW: Convergence to a fixed point of the sequence of Mann type iterates. J. Math. Anal. Appl. 184(1), 75-81 (1994)

11. Rashwan, RA: On the convergence of Mann iterates to a common fixed point or a pair of mappings. Demonstr. Math. XIII(3), 709-712 (1990)

12. Reich, S: Weak convergence theorems for nonexpansive mappings in Banach spaces. J. Math. Anal. Appl. 67, 274-276 (1979)

13. Reich, S, Zaslavski, AJ: Convergence of Krasnoselskii-Mann iterations of nonexpansive operators. Math. Comput. Model. 32(11-13), 1423-1431 (2000)

14. Sharma, S, Deshpande, B: Common fixed point theorems for Mann type iterations. East Asian Math. J. 17(1), 19-32 (2001)

15. Soltuz, SM: The equivalence of Picard, Mann and Ishikawa iterations dealing with quasi-contractive operators. Math. Commun. 10(1), 81-88 (2005)

16. Qihou, L: The convergence theorems of the sequence of Ishikawa iterates for hemicontractive mappings. J. Math Anal. Appl. 148, 55-62 (1990)

17. Liu, L: Fixed points of local strictly pseudo-contractive mappings using Mann and Ishikawa iteration with errors. Indian J. Pure Appl. Math. 26(7), 649-659 (1995)

18. Chidume, CE, Moore, C: Fixed point iteration for pseudocontractive maps. Proc. Am. Math. Soc. 127(4), 1163-1170 (1999)

19. Chidume, CE, Moore, C: Steepest descent method for equilibrium points of nonlinear system with accretive operators. J. Math. Anal. Appl. 245(1), 142-160 (2000)

20. Ibn Dehaish, BA, Khamsi, MA, Khan, AR: Mann iteration process for asymptotic pointwise nonexpansive mappings in metric spaces. J. Math. Anal. Appl. 397(2), 861-868 (2013)

21. Djukić, D, Paunović, L, Radenović, S: Convergence of iterates with errors of uniformly quasi-Lipschitzian mappings in cone metric spaces. Kragujev. J. Math. 35(3), 399-410 (2011)

22. Hussain, N, Rafiq, A: On modified implicit Mann iteration method involving strictly hemicontractive mappings in smooth Banach spaces. J. Comput. Anal. Appl. 15(5), 892-902 (2013)

23. Moore, C: The solution by iteration of nonlinear equations involving Psi-strongly accretive operator in Banach spaces. Nonlinear Anal. 37(1), 125-138 (1999)

24. Moore, C: A double-sequence iteration process for fixed point of continuous pseudocontractions. Comput. Math. Appl. 43, 1585-1589 (2002)

25. Rhoades, BE, Soltuz, SM: The equivalence between the T-stabilities of Mann and Ishikawa iterations. J. Math. Anal. Appl. 318(2), 472-475 (2006)

26. Saddek, AM, Ahmed, SA: On the convergence of some iteration processes for J-pseudomonotone mixed variational inequalities in uniformly smooth Banach spaces. Math. Comput. Model. 46, 557-572 (2007)

27. $\mathrm{Xu}, \mathrm{Y}$ : Ishikawa and Mann iterative process with errors for nonlinear strongly accretive operator equations. J. Math Anal. Appl. 224, 91-101 (1998)

28. Xue, Z: The equivalence of convergence theorems of Ishikawa-Mann iterations with errors for $\Phi$-contractive mappings in uniformly smooth Banach spaces. J. Math. Inequal. 7(3), 477-485 (2013)

29. El-Sayed Ahmed, A, Kamal, A: Some fixed point theorems using compatible-type mappings in Banach spaces. Adv. Fixed Point Theory 4(1), 1-11 (2014)

30. El-Sayed Ahmed, A, Kamal, A: Strong convergence of Mann type doubly sequence iterations with applications. Southeast Asian Bull. Math. 33(1), 1-11 (2009)

31. El-Sayed Ahmed, A, Kamal, A: Fixed points for non-self asymptotically nonexpansive mappings in Banach spaces. Southeast Asian Bull. Math. 34, 201-214 (2010)

32. El-Sayed Ahmed, A, Kamal, A: Construction of fixed points by some iterative schemes. Fixed Point Theory Appl. 2009, Article ID 612491 (2009)

33. Deimling, K: Nonlinear Functional Analysis. Springer, Berlin (1985)

34. Pathak, HK, Khan, MS: Compatible mappings of type (B) and common fixed point theorems of Gregus type. Czechoslov. Math. J. 45(120), 685-698 (1995)

35. Abbas, M, Jovanović, M, Radenović, S, Sretenović, A, Simić, S: Abstract metric spaces and approximating fixed points of a pair of contractive type mappings. J. Comput. Anal. Appl. 13(2), 243-253 (2011)

36. Cholamjiak, P, Cho, YJ, Suantai, S: Composite iterative schemes for maximal monotone operators in reflexive Banach spaces. Fixed Point Theory Appl. 2011, Article ID 7 (2011)

37. Hussain, N, Kumar, V, Kutbi, MA: On rate of convergence of Jungck-type iterative schemes. Abstr. Appl. Anal. 2013 Article ID 132626 (2013)

38. Hussain, N, Rafiq, A, Damjanović, B, Lazović, R: On rate of convergence of various iterative schemes. Fixed Point Theory Appl. 2011, Article ID 45 (2011)

39. Liu, Z, Dong, H, Cho, SY, Kang, SM: Existence and iterative approximations of solutions for certain functional equation and inequality. J. Optim. Theory Appl. 157(3), 716-736 (2013)

10.1186/1687-1812-2014-121

Cite this article as: El-Sayed Ahmed and Ahmed: Fixed points by certain iterative schemes with applications. Fixed Point Theory and Applications 2014, 2014:121 